\title{
PSEUDO-ROTATIONS AND STEENROD SQUARES REVISITED
}

\author{
EGOR SHELUKHIN
}

\begin{abstract}
In this note we prove that if a closed monotone symplectic manifold $M$ admits a Hamiltonian pseudo-rotation, which may be degenerate, then the quantum Steenrod square of the cohomology class Poincaré dual to the point must be deformed. This result gives restrictions on the existence of pseudo-rotations, implying a form of uniruledness by pseudo-holomorphic spheres, and generalizes a recent result of the author. The new component in the proof consists in an elementary calculation with capped periodic orbits.
\end{abstract}

\section{SETuP}

In this paper, $(M, \omega)$ denotes a closed monotone symplectic manifold of dimension $2 n$, with the symplectic form rescaled so that $[\omega]=2 c_{1}(T M)$ on the image of the Hurewicz map $\pi_{2}(M) \rightarrow H_{2}(M ; \mathbb{Z})$. For a Hamiltonian diffeomorphism $\phi \in$ $\operatorname{Ham}(M, \omega)$, we denote by $\operatorname{Fix}(\phi)$ the set of its contractible fixed points, and by $x^{(k)}$ for $x \in \operatorname{Fix}(\phi)$ its image under the inclusion $\operatorname{Fix}(\phi) \subset \operatorname{Fix}\left(\phi^{k}\right)$. Contractible means that the homotopy class $\alpha(x, \phi)$ of the path $\alpha(x, H)=\left\{\phi_{H}^{t}(x)\right\}$ for a Hamiltonian $H \in \mathcal{H} \subset C^{\infty}([0,1] \times M, \mathbb{R})$ generating $\phi$ as the time-one map $\phi_{H}^{1}=\phi$ of its Hamiltonian flow, is trivial]. Here $\mathcal{H}=\cap_{t \in[0,1]} \operatorname{ker}\left(I_{t}\right), I_{t}(H)=\int_{M} H(t,-) \omega^{n}$.

To an isolated fixed point $x$ one associates (cf. [6]) a local cohomology group $H F_{\text {loc }}(\phi, x)$, which is naturally $\mathbb{Z} /(2)$-graded. If we choose a capping $\bar{x}$ of $\alpha(x, H)$, we obtain a $\mathbb{Z}$-graded version, $H F_{\text {loc }}^{*}(H, \bar{x})=H F_{\text {loc }}^{*}(\widetilde{\phi}, \bar{x})$. It depends only on the class $\widetilde{\phi}$ of $\left\{\phi_{H}^{t}\right\}_{t \in[0,1]}$ in the universal cover $\widetilde{\operatorname{Ham}}(M, \omega)$, and the capped orbit $\bar{x}$.

We say that $\phi \in \operatorname{Ham}(M, \omega)$ is an $\mathbb{F}_{2}$ Hamiltonian pseudo-rotation if:

(i) It is perfect, that is for all iterations $k \geq 1$ of $\phi, \operatorname{Fix}\left(\phi^{k}\right)=\operatorname{Fix}(\phi)$. In other words, $\phi$ admits no simple periodic orbits of order $k>1$.

(ii) for all iterations $k \geq 1$,

$$
N\left(\phi^{k}, \mathbb{F}_{2}\right)=\sum_{x \in \operatorname{Fix}(\phi)} \operatorname{dim}_{\mathbb{F}_{2}} H F_{\text {loc }}^{*}\left(\phi^{k}, x^{(k)}\right)=\operatorname{dim}_{\mathbb{F}_{2}} H^{*}\left(M ; \mathbb{F}_{2}\right)
$$

2010 Mathematics Subject Classification. 53D40, 37J10, 37J45.

${ }^{1}$ This class does not depend on the choice of Hamiltonian by a classical argument in Floer theory. 
Remark 1. Observe that a perfect Hamiltonian diffeomorphism necessarily has no symplectically degenerate maxima (see [7]). Furthermore, if $\phi$ is strongly nondegenerate, that is all the points in $\operatorname{Fix}\left(\phi^{k}\right)$ are non-degenerate, for all $k \geq 1$, then $H F_{\text {loc }}(\phi, x) \cong \mathbb{K}$, and all iterations are admissible, that is $\lambda^{k} \neq 1$ for all eigenvalues $\lambda \neq 1$ of $D(\phi)_{x}$. By the Smith inequality in local Floer homology [1, 14], conditions (i) and (ii) imply for iterations $k=2^{m}$, the stronger statement that for all $x \in \operatorname{Fix}(\phi), \operatorname{dim}_{\mathbb{F}_{2}} H F^{\text {loc }}\left(\phi^{k}, x^{(k)}\right)=\operatorname{dim}_{\mathbb{F}_{2}} H F^{\text {loc }}(\phi, x)$. Moreover, [12, Theorem A] indicates that when a Hamiltonian diffeomorphism has a finite number of periodic points, then a condition like (ii) should be satisfied. Showing this in general would bridge the gap between the initial Chance-McDuff conjecture (see for example [7]) and the main result of this note, Theorem A. Dynamics of Hamiltonian pseudo-rotations in higher dimensions were recently studied by Ginzburg and Gürel [5]. We refer thereto for further discussion of this interesting notion, and survey results more closely related to the subject of this paper in Section 2 ,

We remind the reader that the minimal Chern number of $(M, \omega)$ is the index

$$
N=N_{M}=\left[\mathbb{Z}: \text { image }\left(c_{1}(T M): \pi_{2}(M) \rightarrow \mathbb{Z}\right)\right] .
$$

The notion of mean-index, introduced in symplectic topology in [11] is described as follows. For a Hamiltonian $H \in \mathcal{H}$ generating $\widetilde{\phi} \in \widetilde{\operatorname{Ham}}(M, \omega)$ and capped periodic orbit $\bar{x}$ of $H$, we set

$$
\Delta(H, \bar{x})=\Delta\left(\widetilde{\phi}_{H}, \bar{x}\right)=\lim _{k \rightarrow \infty} \frac{1}{k} C Z\left(\widetilde{\phi}^{k}, \bar{x}^{(k)}\right),
$$

where $\bar{x}^{(k)}$ is $\bar{x}$ iterated $k$ times, which is indeed a capped periodic orbit of a Hamiltonian generating $\widetilde{\phi}^{k}$. The limit exists, since the Conley-Zehnder index comes from a quasi-morphism $\widetilde{S p}(2 n, \mathbb{R}) \rightarrow \mathbb{R}$ (see [4]). The mean-index satisfies the following properties that we use below:

(1) homogeneity: $\Delta\left(\widetilde{\phi}^{k}, \bar{x}^{(k)}\right)=k \cdot \Delta(\widetilde{\phi}, \bar{x})$, for all $k \in \mathbb{Z}_{>0}$.

(2) recapping: $\Delta(\widetilde{\phi}, \bar{x} \# A)=\Delta(\widetilde{\phi}, \bar{x})-2\left\langle c_{1}(T M), A\right\rangle, A \in H_{2}^{S}(M ; \mathbb{Z})$.

(3) distance to index: $C Z(\widetilde{\phi}, \bar{x}) \in[\Delta(\widetilde{\phi}, \bar{x})-n, \Delta(\widetilde{\phi}, \bar{x})+n]$,

(4) support of local Floer cohomology: $H F_{\text {loc }}^{r}(\widetilde{\phi}, \bar{x})=0$, unless $r \in[\Delta(\widetilde{\phi}, \bar{x})-$ $n, \Delta(\widetilde{\phi}, \bar{x})+n]$.

(5) symplectically degenerate maxima: by definition, $\bar{x}$ is not a SDM of $\phi$, if $H F_{\text {loc }}^{r}(\widetilde{\phi}, \bar{x})=0$, unless $r \in[\Delta(\widetilde{\phi}, \bar{x})-n, \Delta(\widetilde{\phi}, \bar{x})+n)$.

Remark 2. Observe that, by the symmetry of the Conley-Zehnder index and a simple duality argument, if the reversal $\bar{x}^{(-1)}$ of $\bar{x}$ is not an SDM of $\phi^{-1}$, then $H F_{\text {loc }}^{r}(\widetilde{\phi}, \bar{x})=0$, unless $r \in(\Delta(\widetilde{\phi}, \bar{x})-n, \Delta(\widetilde{\phi}, \bar{x})+n]$. 
Finally, for a quantum cohomology class $\mu \in Q H^{*}\left(M ; \Lambda_{\mathbb{F}_{2}}\right) \backslash\{0\}$ and a Hamiltonian $H \in \mathcal{H}$ with isolated contractible fixed points $\operatorname{Fix}\left(\phi_{H}^{1}\right)$, we recall that the Hamiltonian spectral invariant $c(\mu, H)$ of $\mu$ is carried by a capped 1-periodic orbit $\bar{x}$ of $H$, if in a suitable sense $\bar{x}$ is a lowest action term in a highest minimal action representative of the image $P S S_{H}(\mu)$ of $\mu$ under the PSS isomorphism [10] from the quantum cohomology $Q H^{*}\left(M, \Lambda_{\mathbb{F}_{2}}\right)$ to the filtered Floer cohomology of the Hamiltonian $H$. Keeping in mind the duality between Floer homology and Floer cohomology [8], we refer the reader to [6, 7] for a detailed description of this notion, recording only the following two facts:

(1) spectrality: for $(M, \omega)$ rational, in particular monotone, for each non-zero class $\mu \in Q H^{*}\left(M ; \Lambda_{\mathbb{F}_{2}}\right)$, and $H \in \mathcal{H}$ with $\# \operatorname{Fix}\left(\phi_{H}^{1}\right)<\infty, c(\mu, H)$ is carried by at least one capped 1-periodic orbit $\bar{x}$ of $H$.

(2) contribution to local Floer cohomology: if $\mu$ is homogeneous of degree $k$, and $\bar{x}$ carries $c(\mu, H)$, then $H F_{\text {loc }}^{k}(H, \bar{x}) \neq 0$.

\section{Results}

We call a symplectic manifold strongly uniruled if there exists a non-trivial 3point genus-0 Gromov-Witten invariant $\langle[p t], a, b\rangle_{\beta}$, for $\beta \in H_{2}(M, \mathbb{Z}) \backslash\{0\}$. By [9. Lemma 2.1], if $(M, \omega)$ is not strongly uniruled, then the quantum square $\mu * \mu=0$ for the degree $2 n$ cohomology class $\mu$ Poincaré dual to the point class. A generally different stronger notion than $\mu * \mu=0$, is that the quantum Steenrod square $\mathcal{Q S}(\mu)$, defined in [15], of the volume class $\mu$ satisfy

$$
\mathcal{Q S}(\mu)=h^{2 n} \mu \text {. }
$$

Note that $\mathcal{Q S}(\mu)$ is equal to the classical Steenrod square $S q(\mu)=h^{2 n} \mu$ plus quantum corrections coming from certain pseudo-holomorphic curves. When (1) does not hold, we say that $M$ is $\mathbb{Z} /(2)$-Steenrod uniruled. The main result of this note is the following.

Theorem A. Let $(M, \omega)$ be a closed monotone symplectic manifold admitting an $\mathbb{F}_{2}$ Hamiltonian pseudo-rotation $\phi$. Then $(M, \omega)$ is $\mathbb{Z} /(2)$-Steenrod uniruled.

Remark 3. We observe following [13] that when $(M, \omega)$ is $\mathbb{Z} /(2)$-Steenrod uniruled, then there exists a $J$-holomorphic curve through each point of $M$ for each $\omega$-compatible almost complex structure $J$. Hence, Theorem A provides a geometric obstruction to the existence of pseudo-rotations. Other arguments described in [13] provide obstructions to the existence of pseudo-rotations for monotone manifolds with $N>n$, and rule it out completely for $N>n+1$. We remark that the existence of pseudo-rotations was ruled out in [7] (and references therein), in a strong way, by proving the Conley conjecture, for manifolds such that $\omega(A) \cdot c_{1}(A) \leq 0$ for all spherical homology classes $A \in H_{2}^{S}(M ; \mathbb{Z})$. 
Remark 4. Theorem A was proven by the author in [13] under the additional assumption that $(M, \omega)$ satisfies the Poincaré duality property for Hamiltonian spectral invariants (which holds in particular whenever the minimal Chern number of $(M, \omega)$ satisfies $N>n)$. Under the assumption that $\phi$ is strongly non-degenerate, Theorem $\mathrm{A}$ was also proved by Çineli, Ginzburg, and Gürel in [2], using different additional arguments extending [13. A related, but quite different, result relating the existence of pseudo-rotations to pseudo-holomorphic spheres was shown in [3].

\section{Proof}

The proof of the main result relies on the following observations. First, as mentioned in Remark 1, no fixed point of $\phi$ or $\phi^{-1}$ is a symplectically degenerate maximum (see [7]). In particular, if the capping $\bar{x}$ of a contractible fixed point $x \in \operatorname{Fix}(\phi)$ carries a cohomology class $\mu$ of Conley-Zehnder index $n$ in $H F^{n}(\widetilde{\phi}) \cong Q H^{2 n}\left(M, \Lambda_{\mathbb{F}_{2}}\right)$ for a lift $\widetilde{\phi}$ of $\phi$ to the universal cover $\widetilde{\operatorname{Ham}}(M, \omega)$ of $\operatorname{Ham}(M, \omega)$, then its mean-index $\Delta=\Delta(\widetilde{\phi}, \bar{x})$ satisfies $n<\Delta+n$. Hence

$$
\Delta(\widetilde{\phi}, \bar{x})>0 \text {. }
$$

Second, the following result was proven in [13] specifically in the setting of a pseudo-rotation assuming that (11) holds. Here $\mu \in Q H^{2 n}\left(M, \Lambda_{\mathbb{F}_{2}}\right)$ denotes the cohomology class Poincaré dual to the point.

Theorem B. Let $\psi$ be an $\mathbb{F}_{2}$ pseudo-rotation of $(M, \omega)$ that is not $\mathbb{Z} /(2)$-Steenrod uniruled. Then

$$
c\left(\mu, \widetilde{\psi}^{2}\right) \geq 2 \cdot c(\mu, \widetilde{\psi})
$$

for each $\widetilde{\psi} \in \widetilde{\operatorname{Ham}}(M, \omega)$ covering $\psi$.

We proceed to the proof of the main result, which follows a calculation from [6].

Proof of Theorem $\underline{A}$. Choose $H \in \mathcal{H}$, such that the path $\left\{\phi_{H}^{t}\right\}_{t \in[0,1]}$ represents the class $\widetilde{\phi}$ lifting $\phi$. By the pigeonhole principle, there exists a fixed point $x \in \operatorname{Fix}(\phi)$, and an increasing sequence $k_{i}$ such that $c\left(\mu, H^{\left(r_{i}\right)}\right)$ for $r_{i}=2^{k_{i}}$ is carried by a capping $y_{i}$ of the 1-periodic orbit of the $r_{i}$-iterated Hamiltonian $H^{\left(r_{i}\right)}$ corresponding to $x^{\left(r_{i}\right)}$. By taking a power of $\phi$, we can assume that $r_{1}=1$, and set $y=y_{1}$. Write $y_{i}$ as a recapped iteration of $y$, that is

$$
y_{i}=y^{\left(r_{i}\right)} \# A_{i}
$$

We claim that for $r_{i}$ large, $\omega\left(A_{i}\right) \leq 0$, and $c_{1}\left(A_{i}\right)>0$ contradicting monotonicity. Indeed, write $\mathcal{A}_{i}$ for the action functional of $H^{\left(r_{i}\right)}$, and $\mathcal{A}:=\mathcal{A}_{1}$. Then by (4) and Theorem B.

$$
r_{i} \mathcal{A}(y)-\omega\left(A_{i}\right)=\mathcal{A}_{i}\left(y_{i}\right)=c\left(\mu, H^{\left(r_{i}\right)}\right) \geq r_{i} c(\mu, H)=r_{i} \mathcal{A}(y) .
$$


Hence

$$
\omega\left(A_{i}\right) \leq 0
$$

However, we know that $y_{i}$ carries $c\left(\mu, H^{\left(r_{i}\right)}\right)$, hence $\Delta\left(H^{\left(r_{i}\right)}, y_{i}\right) \in(0,2 n)$ and also $\Delta(H, y) \in(0,2 n)$. Hence $r_{i} \Delta(H, y)>2 n$ for $r_{i}$ large enough, and

$$
2 n>\Delta\left(H^{\left(r_{i}\right)}, y_{i}\right)=r_{i} \Delta(H, y)-2 c_{1}\left(A_{i}\right) \text {. }
$$

Therefore

$$
c_{1}\left(A_{i}\right)>0
$$

\section{ACKNOWLEDGEMENTS}

I thank Paul Seidel for a very useful discussion, and Erman Çineli, Viktor Ginzburg, and Başak Gürel for sharing their preprint [2] with me. At the University of Montréal I was supported by an NSERC Discovery Grant, by the Fonds de recherche du Québec - Nature et technologies, and by the Fondation Courtois.

\section{REFERENCES}

[1] E. Cineli and V. L. Ginzburg. On the iterated Hamiltonian Floer homology. Preprint arXiv:1902.06369, 2019.

[2] E. Cineli, V. L. Ginzburg, and B. Z. Gürel. From pseudo-rotations to holomorphic curves via quantum Steenrod squares. Preprint arXiv:1909.11967, Sept 2019.

[3] E. Cineli, V. L. Ginzburg, and B. Z. Gürel. Pseudo-rotations and holomorphic curves. Preprint arXiv:1905.07567, May 2019.

[4] M. Entov and L. Polterovich. Rigid subsets of symplectic manifolds. Compos. Math., 145(3):773-826, 2009.

[5] V. Ginzburg and B. Gürel. Hamiltonian pseudo-rotations of projective spaces. Invent. Math., Online First:1-50, 2018.

[6] V. L. Ginzburg and B. Z. Gürel. Conley conjecture for negative monotone symplectic manifolds. Int. Math. Res. Not. IMRN, (8):1748-1767, 2012.

[7] V. L. Ginzburg and B. Z. Gürel. Conley conjecture revisited. Int. Math. Res. Not. IMRN, (3):761-798, 2019.

[8] R. Leclercq and F. Zapolsky. Spectral invariants for monotone Lagrangians. J. Topol. Anal., 10(3):627-700, 2018.

[9] D. McDuff. Hamiltonian $S^{1}$-manifolds are uniruled. Duke Math. J., 146(3):449-507, 2009.

[10] S. Piunikhin, D. Salamon, and M. Schwarz. Symplectic Floer-Donaldson theory and quantum cohomology. In Contact and symplectic geometry (Cambridge, 1994), volume 8 of Publ. Newton Inst., pages 171-200. Cambridge Univ. Press, Cambridge, 1996.

[11] D. Salamon and E. Zehnder. Morse theory for periodic solutions of Hamiltonian systems and the Maslov index. Comm. Pure Appl. Math., 45(10):1303-1360, 1992.

[12] E. Shelukhin. On the Hofer-Zehnder conjecture. Preprint, arXiv:1905.04769, May 2019.

[13] E. Shelukhin. Pseudo-rotations and Steenrod squares. Preprint arxiv:1905.05108, May 2019.

[14] E. Shelukhin and J. Zhao. The $\mathbb{Z} /(p)$-equivariant product-isomorphism in fixed point Floer homology. Preprint, arXiv:1905.03666, May 2019.

[15] N. Wilkins. A construction of the quantum Steenrod squares and their algebraic relations. arXiv:1810.02738, 2018. 